\title{
MIMO SAR Using Orthogonal Coding: Design, Performance Analysis, and Verifications
}

\author{
Yueguan Lin,, ${ }^{1,2}$ Yida Fan, ${ }^{1,2}$ Chenglong Jiang, ${ }^{3}$ Zhiqiang Wang, ${ }^{1,2}$ and Weizeng Shao \\ ${ }^{1}$ National Disaster Reduction Center of China, MCA, Beijing 100124, China \\ ${ }^{2}$ Key Laboratory of Disaster Reduction and Emergency Response Engineering of the MCA, Beijing 100124, China \\ ${ }^{3}$ Institute of Electronics, Chinese Academy of Sciences, Beijing 100190, China \\ ${ }^{4}$ Marine Acoustics and Remote Sensing Laboratory, Zhejiang Ocean University, Zhoushan 316000, China
}

Correspondence should be addressed to Yueguan Lin; linyueguan@qq.com

Received 11 March 2015; Accepted 12 May 2015

Academic Editor: Giuseppe Mazzarella

Copyright (C) 2015 Yueguan Lin et al. This is an open access article distributed under the Creative Commons Attribution License, which permits unrestricted use, distribution, and reproduction in any medium, provided the original work is properly cited.

\begin{abstract}
Multiple-input multiple-output (MIMO) synthetic aperture radar (SAR) is a promising technology in radar imaging which provides a better balance of azimuth resolution and swath width compared with traditional single-input single-output (SISO) SAR. It has the potential to help scientists and engineers to design ambitious SAR system with higher resolution and wider swath. This paper studies the principle of MIMO SAR using orthogonal coding waveform and then provides the performance analysis in resolution and swath width. By using orthogonal coding waveform, lower channel interference is obtained, which makes MIMO SAR achieve wider unambiguous range swath and lower azimuth ambiguity. Simulations are carried out by means of the system parameters of real spaceborne SAR platform. A ground-based MIMO SAR imaging system with up and down chirp modulation is also designed. The performances of MIMO SAR and SISO SAR are compared, and the validity and advantage of MIMO SAR are verified.
\end{abstract}

\section{Introduction}

As an active microwave remote sensing imaging tool, synthetic aperture radar (SAR) has the unique capability of obtaining abundant electromagnetic information from ground objects throughout the day and night and in all weather conditions. As such, it has been widely used in military reconnaissance, mapping, resource exploration, environmental protection, disaster emergency and management, and so forth. Two most important characteristics of modern SAR systems-azimuth resolution and swath coverage-are contradictive in system design. In order to improve the azimuth resolution and swath width, people have done a lot of research. ScanSAR is a traditional technique to acquire unambiguous wide swath SAR image, while it reduces the image resolution [1]. Spotlight obtains very fine resolution while it cannot observe the earth continuously in azimuth [2]. Other techniques including wide swath imaging via elevation and azimuth multiple beams $[3,4]$ or azimuth digital beam forming [5-7] and high resolution wide swath
(HRWS) imaging via multidimensional waveform encoding are proposed [8], while they cause the system complexity ascension, reliability reduction, or signal processing difficulty increasing.

Since multiple-input and multiple-output (MIMO) radar system has enormous potential to improve the performance of the radar system, MIMO radar technology continues to be the research hotspot in recent years [9-11]. More attention is paid to MIMO SAR technology [12-16]. MIMO SAR transmits and receives signals via multiple antennas in azimuth and/or range at the same time. For each receiving channel of MIMO SAR, it divides echoes transmitted from different channels before imaging processing.

In this paper, MIMO SAR imaging based on orthogonal coding waveforms is proposed, and simulation and experiments in an anechoic chamber are carried out. MIMO SAR system increases the number of effective phased centers, acquires more scene information, and has the ability to obtain high resolution and wide swath. In order to separate signals 


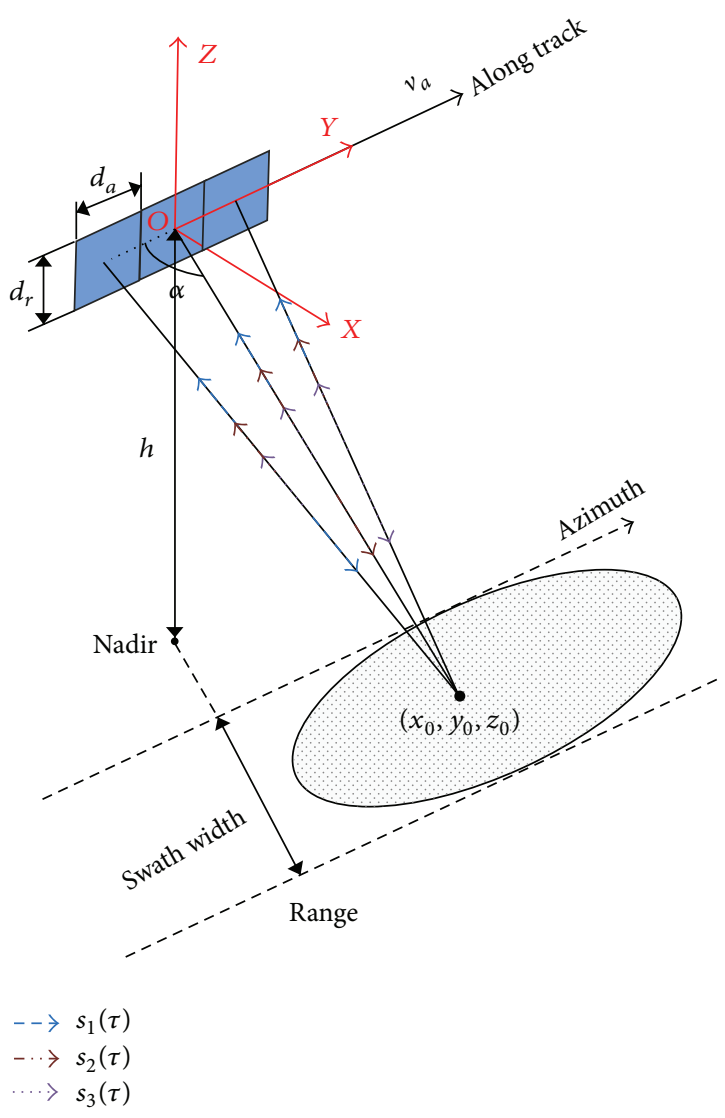

FIGURE 1: MIMO SAR geometric model.

from different channels in receivers, transmitted waveforms and signal processing methods have to be carefully designed.

\section{MIMO SAR Using Orthogonal Waveform}

There are different antenna arrangements for MIMO SAR system. In this work, azimuth linear uniform array configuration with each subantenna both for transmitting signals and receiving echoes is considered, which is shown in Figure 1. MIMO SAR system transmits quadrature encoder waveform and receives echoes using multiple subantennas in azimuth. After MIMO SAR system's subantenna receiving echoes, signals from different emission channel are separated by match filtering. According to the principle of equivalent phase center, MIMO SAR achieves more effective phase centers in azimuth comparing to traditional single-input single-output (SISO) SAR. When the same PRF is adapted, MIMO SAR with $N$ antennas which are equally spaced in azimuth can achieve $2 \mathrm{~N}-1$ times effective samples in a pulse repetition interval (PRI). Therefore, when the same azimuth resolution was maintained, PRF of MIMO SAR system can be reduced to $2 N-1$ of SISO SAR. When the same pulse repetition frequency (PRF) is adapted, MIMO SAR will have greater Doppler bandwidth and without the azimuth ambiguity caused by azimuth undersampling, which can achieve higher azimuth resolution and wider swath at the same time.
Geometric model of MIMO SAR is illustrated in Figure 1. The platform flight speed is $v_{a}$ and platform height is $h$. The coordinate system is established by setting the geometric center antenna array as the origin. $y$-axis represents along track direction, $z$-axis is opposite to the direction of earth center, and $x$-axis is perpendicular to the $y-z$ plane. $t$ represents the time. The fast time is $\tau=t-m T$, and the sampled slow time is represented as $t_{m}=m T$, where $m$ is an integer and $T$ is equal to PRI. As the system has $N$ subantennas for both transmitting and receiving signals, the $n$th baseband quadrature encoded signal transmitted by $n$th is $p_{n}(\tau), n=1, \ldots, N$, and these encoded signals satisfy the following equation:

$$
\int p_{n}(\tau) p_{u}^{*}(-\tau) d \tau= \begin{cases}1, & n=u, \\ c_{n u}, & n \neq u,\end{cases}
$$

where the upper symbol $*$ stands for complex conjugation and $c_{n u}(n \neq u)$ is the cross-correlation between $p_{n}(\tau)$ and $p_{u}(\tau)$. For the ideal orthogonal signals, $c_{n u}=0(n \neq u)$. These signals are hardly ideal and orthogonal in real applications, but they can be designed as suboptimally orthogonal as possible. The signal transmitted in the $n$th channel after modulation can be expressed as

$$
\begin{aligned}
& s_{n}(\tau)=\operatorname{rect}\left(\frac{\tau}{T_{p}}\right) \exp \left(j 2 \pi f_{c} \tau\right) p_{n}(\tau), \\
& n=1,2, \ldots, N,
\end{aligned}
$$

where $T_{p}$ is the duration of pulse and $f_{c}$ is carrier frequency. According to system geometry shown in Figure 1, the shortest slant range between target point located at $\left(x_{0}, y_{0}, z_{0}\right)$ and platform is $R_{0}=\sqrt{x_{0}^{2}+h^{2}}$. The distance between the $n$th channel and target at $t_{m}=m T$ is

$$
R_{n}\left(t_{m}\right)=\sqrt{\left(y_{0}-v_{a} t_{m}-n d_{a}\right)^{2}+R_{0}^{2}},
$$

where $d_{a}$ is the distance between two phase centers of adjacent antennas. The signals received by the $n$th channel are the echoes from all objects illuminated by $N$ transmitted waveforms. The received signals after removing highfrequency components can be represented as

$$
\begin{aligned}
& r_{n}\left(t_{m}, \tau\right)=\sum_{u=1}^{N} \sigma \\
& \cdot \operatorname{rect}\left\{\frac{\tau-R_{u, n}\left(t_{m}\right) / C}{T_{p}}\right\} p_{n}\left\{\tau-\frac{R_{u, n}\left(t_{m}\right)}{C}\right\} \\
& \cdot \operatorname{rect}\left\{\frac{Y_{n}+v_{a} t_{m}-y_{0}}{L_{a}}\right\} \exp \left\{-\frac{j 2 \pi R_{u, n}\left(t_{m}\right)}{\lambda}\right\},
\end{aligned}
$$

where $\sigma$ is target scattering coefficient, $C$ is the electromagnetic wave propagation velocity in vacuum, $L_{a}$ is the length of synthetic aperture, $\operatorname{rect}(\cdot)$ represents rectangular function, and $R_{u, n}\left(t_{m}\right)$ is two-way distance with the $u$ th channel transmitter and the $n$th channel receiver at $t_{m}$ :

$$
R_{u, n}\left(t_{m}\right)=R_{u}\left(t_{m}\right)+R_{n}\left(t_{m}\right) .
$$


$Y_{n}$ is along track coordinate of the $n$th channel at $t=0$ :

$$
Y_{n}=\frac{(2 n-N-1) d_{a}}{2}, \quad n=1, \ldots, N
$$

To distinguish the signals transmitted from different antennas, match filtering after signals received is adapted. The component of echoes from $u$ th subantenna can be achieved by match filtering using the $u$ th waveform:

$$
\begin{aligned}
r_{u, n}\left(t_{m}, \tau\right)= & \sigma \cdot \operatorname{psf}_{u}\left(\tau-\frac{R_{u, n}}{C}\right) \\
& \cdot \operatorname{rect}\left\{\frac{\left(Y_{n}+Y_{u}\right) / 2+v_{a} t_{m}-y_{0}}{L_{a}}\right\} \\
& \cdot \exp \left\{-\frac{j 2 \pi R_{u, n}\left(t_{m}\right)}{\lambda}\right\},
\end{aligned}
$$

where $\operatorname{psf}_{u}=F^{-1}\left(\left|P_{u}(f)\right|\right), P_{u}(f)=F\left(s_{u}(\tau)\right), F(\cdot)$ indicates Fourier transform, and $F^{-1}(\cdot)$ indicates inverse Fourier transform. In (7), the mismatch energy caused by nonideal orthogonality among transmitted waveforms is ignored. For the sake of simplicity, we ignore the range migration correction and the inconsistency among different channels which can be compensated in real situation. According to the principle of equivalent phase center, the echoes components of the $u$ th channel transmitted and the $n$th channel received are equivalent to that of the $n$th channel transmitted and the $n$th channel received, and only a phase difference changing with the observed slant distance and look angle exists which can also be compensated. So (7) can be simplified to

$$
\begin{aligned}
r_{u, n}\left(t_{m}, \tau\right)= & \sigma \cdot \operatorname{psf}_{u}\left(\tau-\frac{R_{u, n}}{C}\right) \\
& \cdot \operatorname{rect}\left\{\frac{Y_{u, n}+v_{a} t_{m}-y_{0}}{L_{a}}\right\} \\
& \cdot \exp \left\{-\frac{j 4 \pi R_{u, n}^{\prime}\left(t_{m}\right)}{\lambda}\right\},
\end{aligned}
$$

where

$$
\begin{aligned}
R_{u, n}^{\prime}\left(t_{m}\right) & =\sqrt{\left(y_{0}-v_{a} t_{m}-Y_{u, n}\right)^{2}+R_{0}^{2}}, \\
Y_{u, n} & =(u+n-1-N) d_{a} .
\end{aligned}
$$

The phase error caused by this approximation can be calculated as follows:

$$
\begin{aligned}
& \Delta \varphi_{u, n}=\frac{2 \pi}{\lambda}\left(2 R_{u, n}^{\prime}-R_{u, n}\right) \\
& =\frac{2 \pi}{\lambda}\left\{2 \sqrt{\left(y_{0}-v_{a} t_{m}-Y_{u, n}\right)^{2}+R_{0}^{2}}\right. \\
& -\sqrt{\left(y_{0}-v_{a} t_{m}-Y_{u}\right)^{2}+R_{0}^{2}} \\
& \left.-\sqrt{\left(y_{0}-v_{a} t_{m}-Y_{n}\right)^{2}+R_{0}^{2}}\right\}=-\frac{\pi(n-u)^{2} d_{a}^{2}}{2 \lambda R_{0}} \\
& \cdot \sin \alpha+O\left(\frac{\pi(n-u)^{2} d_{a}^{2}}{2 \lambda R_{0}}\right),
\end{aligned}
$$

where $\alpha$ is looking angle. After range match filtering of the echoes of $N$ channels with $N$ orthogonal waveforms, $N^{2}$ signals corresponding to $N^{2}$ effective phase centers are achieved. Due to spatial relationship among $N$ channels, some effective phase centers are coincident. Through optimized design of spatial distribution of channels, more equivalent phase centers can be obtained. Since the distance between all the adjacent channels is the same in this paper, the number of effective equivalent phase centers equals $2 N-1$. For the antennas configuration shown in Figure 1, the following equation holds via selecting an appropriate PRF:

$$
\frac{v_{a}}{\mathrm{PRF}}=\frac{N d_{a}}{2} \text {. }
$$

This means SAR platform moves just one half of its total antenna length between subsequent radar pulses. When this condition is satisfied, the equivalent phase centers are equally spaced along azimuth after range match filtering and the distance between two adjacent equivalent phase centers is $d_{a} / 2$. Equation (8) can be expressed as follows:

$$
\begin{aligned}
\xi_{q}\left(t_{m}, \tau\right)= & \sigma \cdot \operatorname{psf}_{u}\left(\tau-\frac{R_{q}}{C}\right) \\
& \cdot \operatorname{rect}\left\{\frac{Y_{q}+v_{a} t_{m}-y_{0}}{L_{a}}\right\} \exp \left\{-\frac{j 4 \pi R_{q}}{\lambda}\right\},
\end{aligned}
$$

where

$$
\begin{aligned}
& R_{q}=\sqrt{\left(y_{0}-v_{a} t_{m}-Y_{q}\right)^{2}+R_{0}^{2}}, \\
& q=1,2, \ldots, 2 N-1, \\
& Y_{q}=\frac{(2 q-N-1) d_{a}}{2}, \quad q=1, \ldots, 2 N-1 .
\end{aligned}
$$

After the range compressed signals are rearranged according to azimuth chronological order, the azimuth focusing is achieved via traditional SISO SAR technology. MIMO SAR processing flowchart from signals receiving to high resolution and wide swath image focusing is illustrated in Figure 2. 


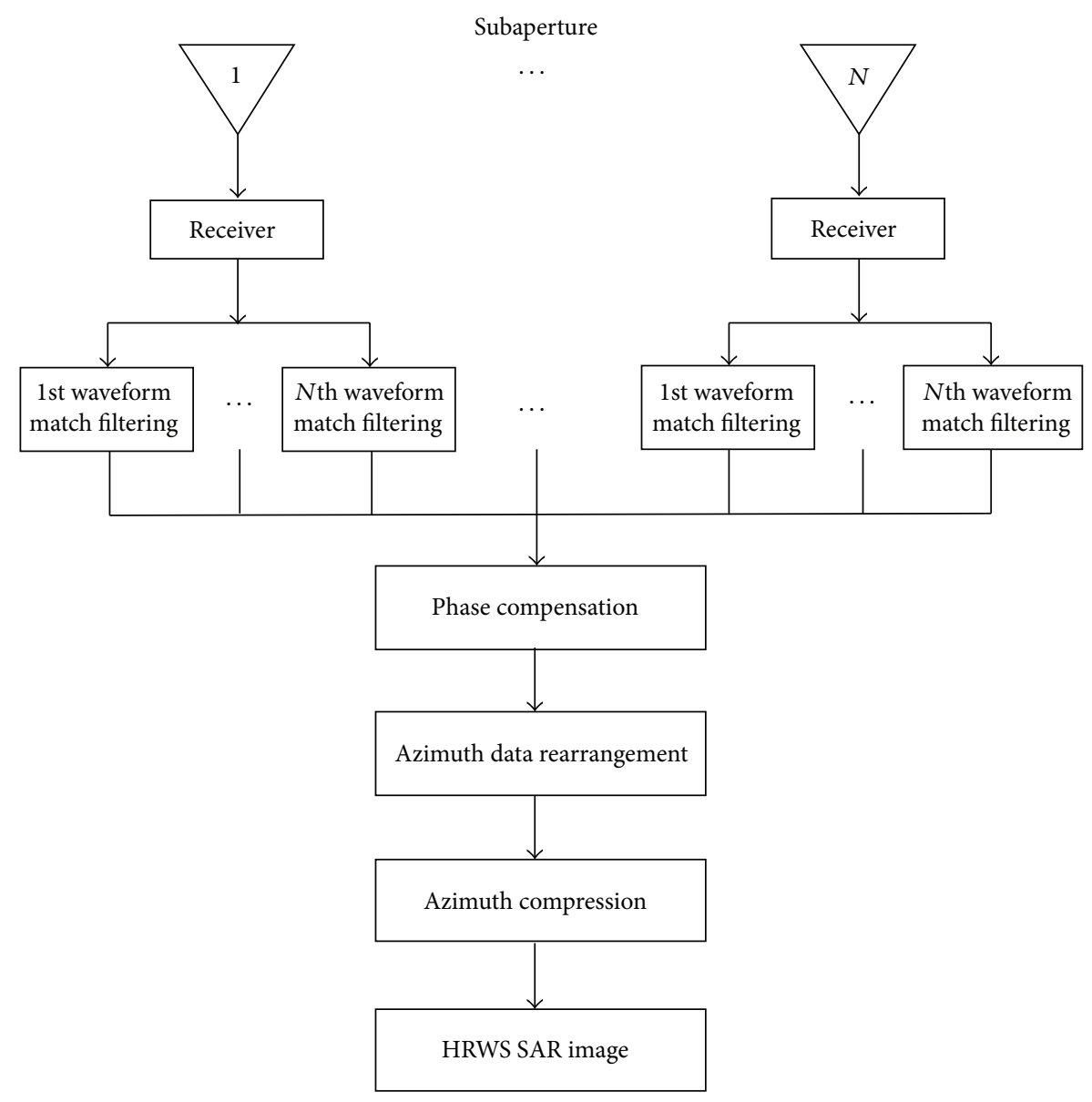

FIGURE 2: MIMO SAR processing flowchart.

\section{HRWS SAR Simulation}

In this part, restrictions between swath width and azimuth resolution for SISO and MIMO SAR are analyzed and simulated.

For SAR system parameters design, the selection of PRF is restricted by the trade-off of swath width and azimuth resolution. On the one hand, echoes from the observed swath should arrive at receiver in one PRI after one or multiple PRIs, which can be expressed as follows:

$$
\begin{aligned}
& \frac{2 R_{\min }}{C} \geq \frac{i-1}{\mathrm{PRF}}, \\
& \frac{2 R_{\max }}{C} \leq \frac{i}{\mathrm{PRF}},
\end{aligned}
$$

where $i$ is an arbitrary positive integer and $R_{\min }$ and $R_{\max }$ represent the nearest and farthest distance between swath and receiver, respectively. By combining (14) and (15),

$$
\frac{(i-1) C}{2 R_{\min }} \leq \mathrm{PRF} \leq \frac{i C}{2 R_{\max }} .
$$

On the other hand, in order to avoid nadir echo interference, the echoes' receiving window is constrained in the duration between two echoes from nadir:

$$
\begin{aligned}
& \frac{2 R_{\min }}{C} \geq \frac{2 h}{C}+\frac{i-1}{\mathrm{PRF}}, \\
& \frac{2 R_{\max }}{C} \leq \frac{2 h}{C}+\frac{i}{\mathrm{PRF}} .
\end{aligned}
$$

Equation (17) can be simplified as

$$
\frac{(i-1) C}{2\left(R_{\min }-h\right)} \leq \mathrm{PRF} \leq \frac{i C}{2\left(R_{\max }-h\right)}
$$

The azimuth resolution $\delta_{a}$ is deduced as follows:

$$
\begin{aligned}
& B_{a}=\frac{2 v_{a}}{d_{a}}=\frac{v_{a}}{\delta_{a}}, \\
& \delta_{a}=\frac{v_{a}}{B_{a}}=k_{a} \frac{v_{a}}{\mathrm{PRF}},
\end{aligned}
$$




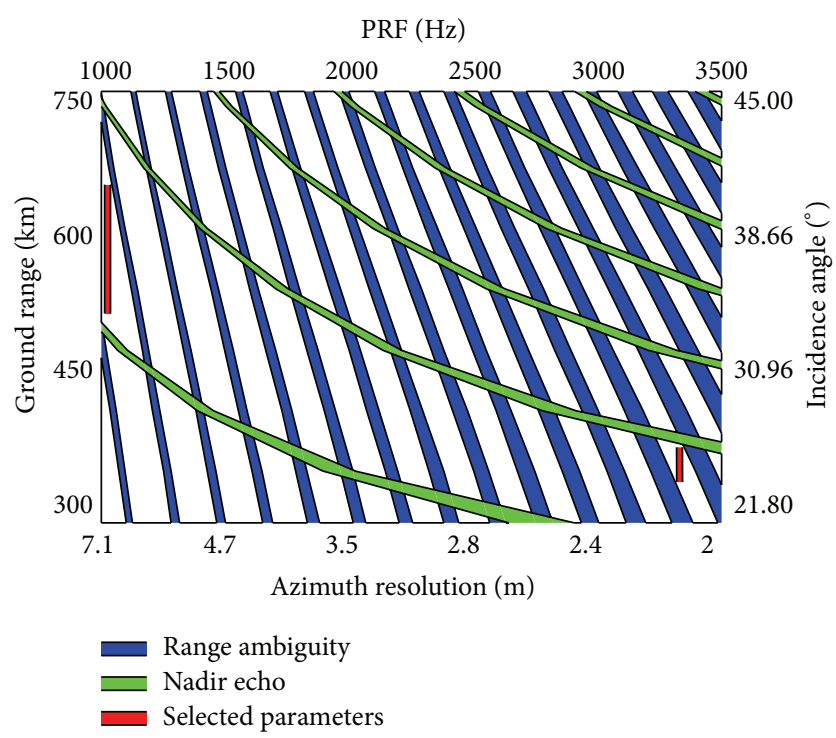

FIGURE 3: Swath width and azimuth resolution restriction for SAR system design.

where $k_{a}$ is azimuth sampling factor. It can be seen that $\delta_{a}$ is inversely proportional to PRF. The constraint condition of azimuth resolution $\delta_{a}$ can be expressed as

$$
\begin{gathered}
\frac{2 k_{a} v_{a} R_{\max }}{i C} \leq \delta_{a} \leq \frac{2 k_{a} v_{a} R_{\min }}{(i-1) C}, \\
\frac{2 k_{a}\left(R_{\max }-h\right)}{i C} \leq \delta_{a} \leq \frac{2 k_{a}\left(R_{\min }-h\right)}{(i-1) C} .
\end{gathered}
$$

To compare the SISO SAR and MIMO SAR system difference in swath width and azimuth resolution when other parameters are identical, a simulation is carried out. The system parameters of a real spaceborne platform and system are listed in Table 1. Swath width and azimuth resolution restriction according to (20) are shown in Figure 3. As can be seen, for SISO SAR, the azimuth resolution becomes low when the swath width is set large and vice versa. For SISO $\mathrm{SAR}$, if the expected slant range resolution is $2 \mathrm{~m}$ and the azimuth resolution is $2.2 \mathrm{~m}$, the swath width achieved is about $60 \mathrm{~km}$. For comparison, MIMO SAR system has two channels for both transmitting and receiving signals. The transmitted waveforms are two chirps having the same bandwidth, and one is up chirp modulated and the other is down chirp modulated. As MIMO SAR system's effective azimuth phase centers are three times as that of SISO SAR system, when identical effective azimuth sampling rate (i.e., effective PRF) is set, MIMO SAR's system PRF is only just one-third of SISO SAR's system PRF. To keep effective PFR of $3150 \mathrm{~Hz}$, MIMO SAR only needs system PRF of $1050 \mathrm{~Hz}$. This lower system PRF of MIMO SAR achieves a much wider unambiguous swath. In our simulation, when the same azimuth resolution is kept as $2.2 \mathrm{~m}$, MIMO SAR has $180 \mathrm{~km}$ swath width which is three times wider than that of SISO SAR.

\begin{tabular}{|c|c|c|}
\hline Working mode & SISO & MIMO \\
\hline Transmit signal & Up chirp & Up/down chirp \\
\hline Platform height $(\mathrm{km})$ & 750 & 750 \\
\hline Platform velocity (m/s) & 7090 & 7090 \\
\hline Carrier wavelength (m) & 0.031 & 0.031 \\
\hline Pulse bandwidth (MHz) & 75 & 75 \\
\hline $\operatorname{PRF}(\mathrm{Hz})$ & 3150 & 1050 \\
\hline Effective PRF (Hz) & 3150 & 3150 \\
\hline Slant range resolution $(\mathrm{m})$ & 2 & 2 \\
\hline Azimuth resolution (m) & 2.2 & 2.2 \\
\hline Swath width (km) & 60 & 180 \\
\hline Ground range $(\mathrm{km})$ & {$\left[\begin{array}{ll}330 & 390\end{array}\right]$} & {$\left[\begin{array}{ll}510 & 690\end{array}\right]$} \\
\hline Incident angle $\left(^{\circ}\right)$ & {$\left[\begin{array}{ll}23.7 & 27.5\end{array}\right]$} & {$\left[\begin{array}{ll}34.2 & 42.6\end{array}\right]$} \\
\hline
\end{tabular}

TABLE 1: Simulation SAR system parameters.

TABLE 2: Ground-based MIMO SAR system parameters.

\begin{tabular}{lc}
\hline Parameter & Value \\
\hline Number of antennas & 4 \\
Transmitted signal & Up/down chirp \\
Antenna azimuth distance $\left(R_{a}\right)$ & $20 \mathrm{~cm}$ \\
Antenna elevation distance $\left(R_{r}\right)$ & $18.8 \mathrm{~cm}$ \\
Bandwidth & $500 \mathrm{MHz}$ \\
Carrier frequency & $8 \mathrm{GHz}$ \\
Pulse duration & $1 \mu \mathrm{S}$ \\
Platform velocity & $1 \mathrm{~cm} / \mathrm{s}$ \\
Shortest slant range & $215.8 \mathrm{~cm}$ \\
Azimuth movement interval & $6 \mathrm{~cm}$ \\
Azimuth span & $-54 \mathrm{~cm}-+54 \mathrm{~cm}$ \\
\hline
\end{tabular}

\section{MIMO SAR Using Orthogonal Coding Waveforms Experiment}

4.1. Experimental Scheme. A ground-based experimental system is designed to verify the MIMO SAR's feasibility and advantage. MIMO SAR experimental system is composed of four horn antennas, rail system, arbitrary waveform generator, oscilloscope, cables, and remote control port, which are shown in Figure 4. Remote control port sends commands to the controller, and the controller drives the motor of orbital platform so that the antennas can be moved in the track which simulates the movement of MIMO SAR platform. Arbitrary waveform generator can generate the desired waveform whose bandwidth, wave duration, power for adapted chirp signal (i.e., linear frequency modulation signal), and chirp rate can be set. Arbitrary waveform generator transmits the selected waveforms to transmit antennas through waveguide. Other two antennas receive the echoes and transmit them to oscilloscope, and the oscilloscope can display and save the sampled echo signal in real time. Platform and system parameters used in the experiment are shown in Table 2.

In the system, two antennas are for transmitting signals and two for receiving echoes. Since the arbitrary waveform generator can only emit one signal at a time, we realize multiple inputs and multiple outputs via time division in 


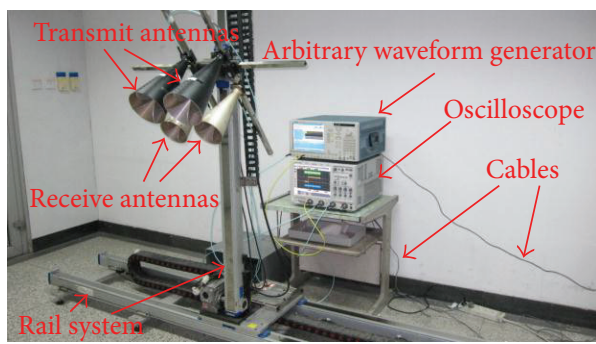

(a) Experimental configuration

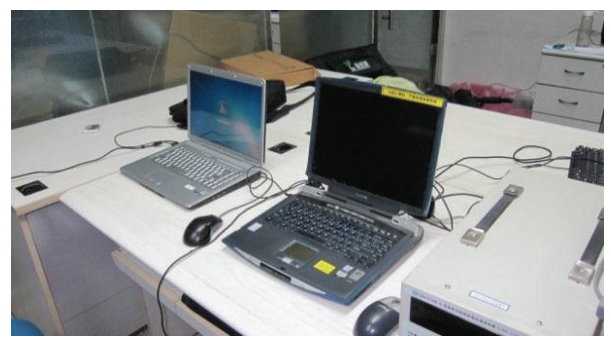

(c) Remote control port

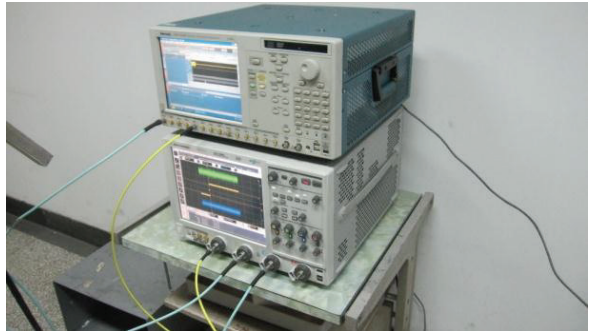

(b) Arbitrary waveform generator and oscilloscope

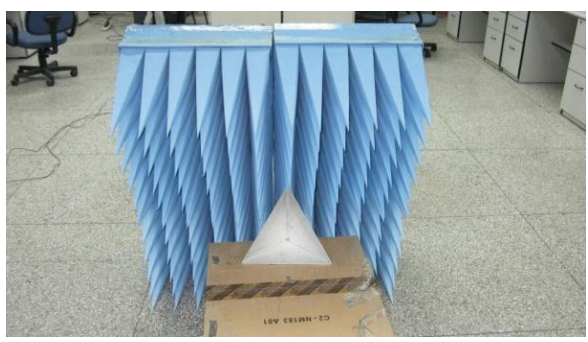

(d) Triangle reflector

FIGURE 4: MIMO SAR experimental configuration and observed target.

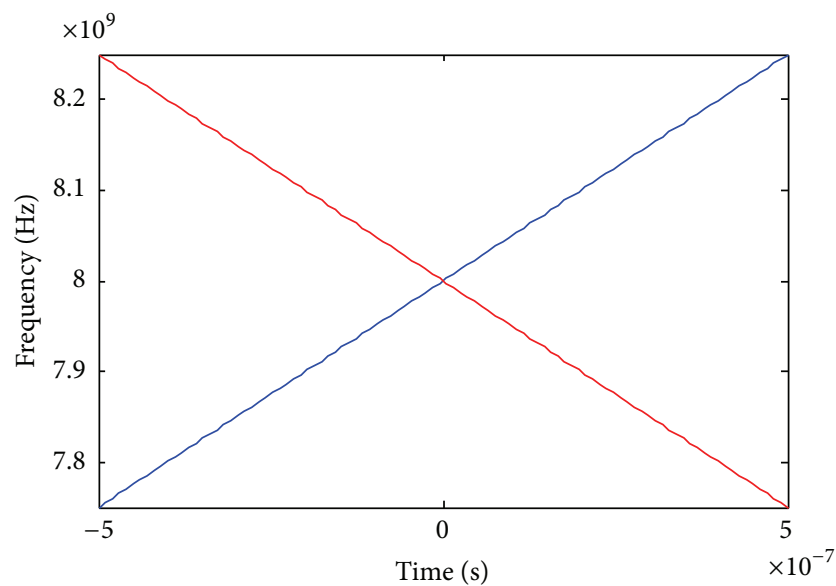

- Up modulated chirp

— Down modulated chirp

FIGURE 5: Transmitted chirps.

two steps. Firstly we use the up-right (as shown in Figure 4) antenna to transmit up chirp signal and below two channels to receive echoes at the same time. Secondly we use the up-left antenna to transmit down chirp signal and below two channels to receive echoes. Platform moving trajectories are identical twice. After these operations, samples received by the same antenna are superimposed into one group respectively. At this point the desired MIMO SAR samples are acquired.

4.2. Data Processing. The transmitted chirp signals are shown in Figure 5. The normalized autocorrelation and crosscorrelation of two transmitted chirp signals are shown in
Figure 6. The autocorrelation is the standard point target response, while the cross-correlation is a uniform energy distribution in the whole time duration. This cross-correlation energy will cause ambiguity in the focused MIMO SAR image. For scenes consisting of discrete targets, the level of ambiguity is low and can be neglected. However, for scenes with extended targets, the SAR image will be seriously blurred. To solve this problem, scientists and engineers have done a lot of beneficial research and experiments on antenna configuration and signal processing and have built solid foundation for the engineering realization of MIMO SAR [17-21]. After oscilloscope sampling, we get the baseband data of echoes via quadrature demodulation and obtain I and $\mathrm{Q}$ signals. As the range sampling rate determined by oscilloscope is $40 \mathrm{GHz}$ which is much higher than signal bandwidth, we resample the echoes to $1 \mathrm{GHz}$ of bandwidth.

For the echoes received by antenna $A_{1}$ as shown in Figure 7, we use up chirp and down chirp to do match filtering, and for echoes received by antenna $A_{2}$ we use up chirp to do match filtering. According to displaced phase center antenna (DPCA) technique, the geometric midpoint of transmitted antenna phase center and received antenna phase center is defined as the effective phase center for each sample, as shown in Figure 7. This definition causes phase difference, as the distance of real electromagnetic wave propagation path is different from the two-way distance from effective center to target. After range compression, this difference needs to be compensated. Through three-dimensional space geometric calculation, the distance of different transceiver combination can be expressed as

$$
\begin{aligned}
R_{23}(t) & =R_{14}(t) \\
& =\sqrt{\left(\frac{R_{a}}{2}+R_{0} \tan (\alpha(t))\right)^{2}+\left(\frac{R_{r}}{2}\right)^{2}+R_{0}^{2}}
\end{aligned}
$$




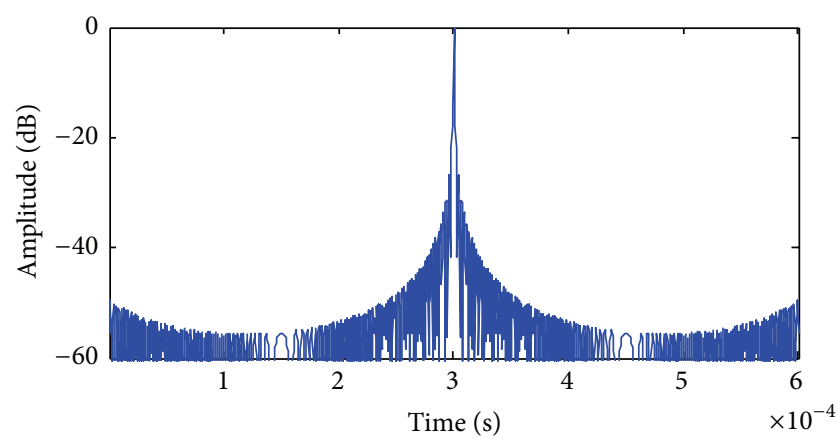

(a) Autocorrelation of up modulated chirp

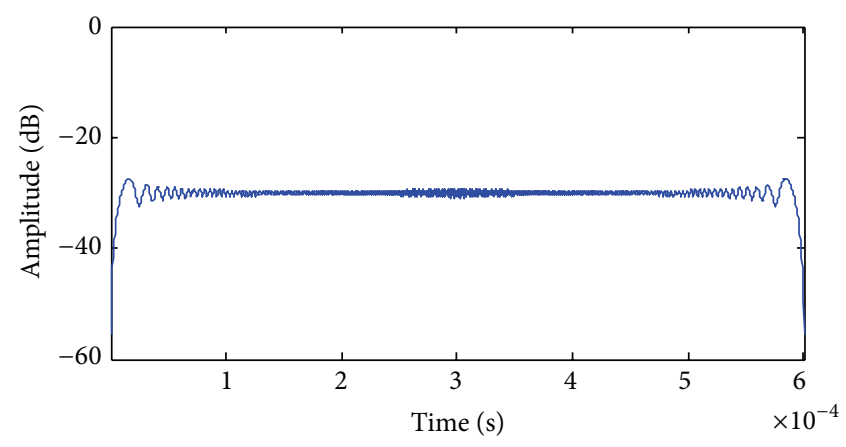

(b) Cross-correlation between up modulated chirp and down modulated chirp

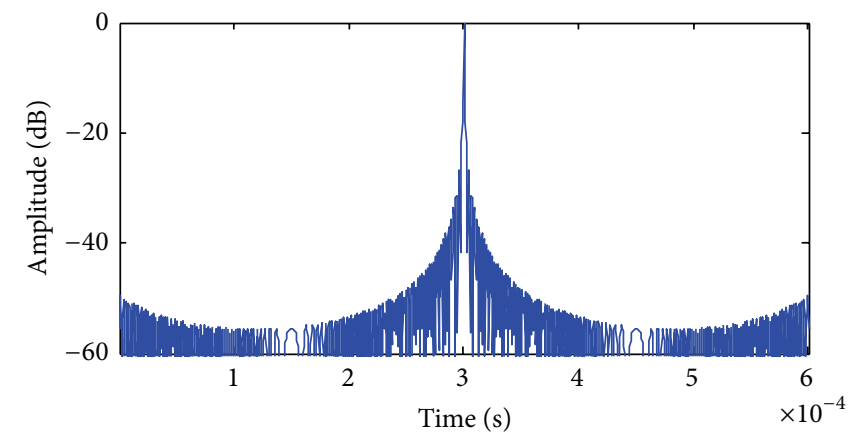

(c) Self-correction of down modulated chirp

FIGURE 6: Correction between transmitted chirp signals.

$$
\begin{aligned}
& +\sqrt{\left(\frac{R_{a}}{2}-R_{0} \tan (\alpha(t))\right)^{2}+\left(\frac{R_{r}}{2}\right)^{2}+R_{0}^{2}}, \\
R_{13}(t)= & R_{1}(t)+R_{3}(t) \\
= & 2 \sqrt{\left(\frac{R_{a}}{2}+R_{0} \tan (\alpha(t))\right)^{2}+\left(\frac{R_{r}}{2}\right)^{2}+R_{0}^{2}}, \\
R_{24}(t)= & R_{2}(t)+R_{4}(t) \\
= & 2 \sqrt{\left(\frac{R_{a}}{2}-R_{0} \tan (\alpha(t))\right)^{2}+\left(\frac{R_{r}}{2}\right)^{2}+R_{0}^{2}} .
\end{aligned}
$$

The deduction of above three equations is under the condition that the plane determined by lines connecting target and effective centers is perpendicular to the plane determined by phase centers of four antennas. The two-way distance from effective centers to target can be expressed as follows:

$$
\begin{aligned}
\widetilde{R}_{23} & =\widetilde{R}_{14}(t)=2 \frac{R_{0}}{\cos (\alpha(t))}, \\
\widetilde{R}_{13}(t) & =2 \sqrt{\left(\frac{R_{a}}{2}+R_{0} \tan (\alpha(t))\right)^{2}+R_{0}^{2}}, \\
\widetilde{R}_{24}(t) & =2 \sqrt{\left(\frac{R_{a}}{2}-R_{0} \tan (\alpha(t))\right)^{2}+R_{0}^{2}} .
\end{aligned}
$$

The corresponding equivalent phase differences are

$$
\begin{aligned}
& \Delta \phi_{23}(t)=\Delta \phi_{14}(t)=\frac{2 \pi}{\lambda}\left(R_{23}(t)-\widetilde{R}_{23}\right), \\
& \Delta \phi_{13}(t)=\frac{2 \pi}{\lambda}\left(R_{13}(t)-\widetilde{R}_{13}\right) \\
& \Delta \phi_{24}(t)=\frac{2 \pi}{\lambda}\left(R_{24}(t)-\widetilde{R}_{24}\right) .
\end{aligned}
$$

These phase differences as a function of azimuth sampling position are shown in Figure 8. It can be found that equivalent phase differences of transmit-receive antennas in vertical planes $\Delta \phi_{13}$ and $\Delta \phi_{24}$ are close to 0.7 , and the equivalent phase differences of transmit-receive antennas not in vertical planes $\Delta \phi_{23}$ and $\Delta \phi_{14}$ are about 1.4. Comparing to equivalent phase differences illustrated in [17] which are small to negligible, equivalent phase differences in this experiment are comparatively large. Reason for this difference is that for airborne and spaceborne SAR system, the ratio between length synthetic aperture and distance from platform to target is about $1 / 100$, while the ratio for this experiment is about $1 / 2$. To avoid the ambiguity after azimuth compression, these equivalent phase differences need to be compensated.

After rearranging the range compressed data with the same azimuth sampling space, range migration correction and azimuth compression are carried out. Azimuth and range slices of imaging result are shown in Figure 9. We can find that the target is finely compressed. The ambiguity at range about $-4.5 \mathrm{~m}$ is caused by leakage of the direct wave. 




FIGURE 7: Geometry of equivalent beam phase centers.

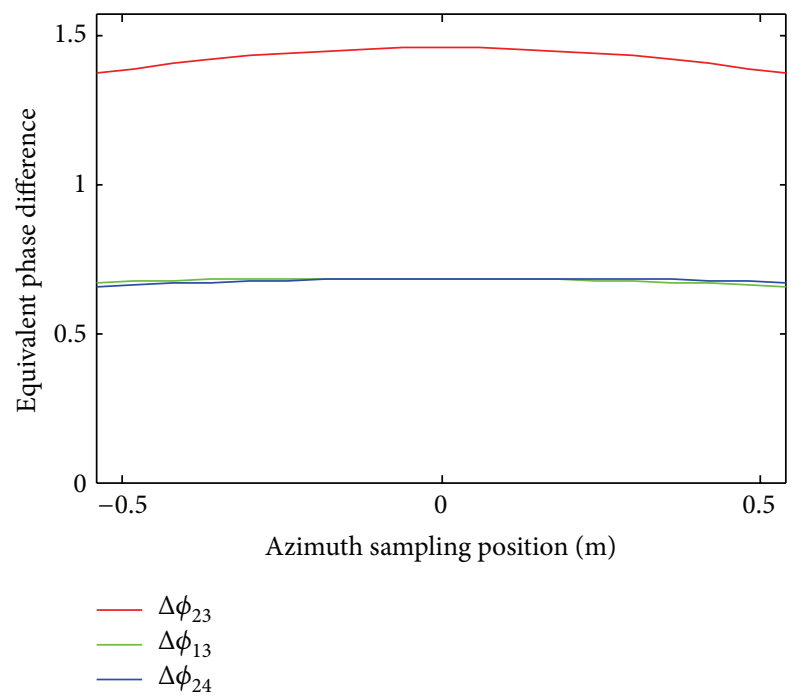

FIgURE 8: Equivalent phase difference as a function of azimuth sampling position.

Main lobe $(\mathrm{ML})$ width on the $-3 \mathrm{~dB}$ level, peak side lobe ratio (PSLR), and integrated side lobe ratio (ISLR) are commonly used to measure SAR imaging quality. These three indictors of MIMO SAR imaging result are listed in Table 3. The theoretical azimuth resolution is $3.2 \mathrm{~cm}$ and the theoretical range resolution is $0.886 C / 2 B_{r}=26.6 \mathrm{~cm}$. We find that both azimuth real resolution and range real resolution are close to theoretical resolutions.

We set the same system parameters as shown in Table 2 for SISO SAR with one transmitter and one receiver channel. Azimuth and range slices of imaging result are shown in Figure 10, and the performance indexes are shown in Table 4.
TABLE 3: MIMO SAR performance for imaging results.

\begin{tabular}{lccc}
\hline & IRW $(\mathrm{cm})$ & PSLR $(\mathrm{dB})$ & ISLR $(\mathrm{dB})$ \\
\hline Azimuth & 3.9 & -17.5 & -12.2 \\
Range & 26.4 & -11.1 & -6.9 \\
\hline
\end{tabular}

TABLE 4: SISO SAR performance for imaging results.

\begin{tabular}{lccc}
\hline & IRW $(\mathrm{cm})$ & PSLR $(\mathrm{dB})$ & ISLR $(\mathrm{dB})$ \\
\hline Azimuth & 5.3 & -2.3 & 1.7 \\
Range & 26.9 & -12.0 & -7.4 \\
\hline
\end{tabular}

We find that the result is strongly blurred in azimuth which is caused by azimuth undersampling.

4.3. Discussion. From the imaging results shown above, we find that SISO SAR system suffers from azimuth ambiguity, while MIMO SAR with the same system parameters is free of ambiguity. Azimuth sampling rate MIMO SAR is three times as much as SISO SAR with the same system parameters and PRF. If they keep the same equivalent sampling rate, MIMO SAR only needs one-third of sampling rate as SISO SAR, which means, with the same azimuth resolution, MIMO SAR can reach 3 times wider swath than that of SISO SAR.

Although digital simulation and ground experiment verify the effectiveness of MIMO SAR using orthogonal coding waveform, there are several challenges needed to be conquered in the design of MIMO SAR system. First, the orthogonal coding waveforms should be designed as suitable for MIMO SAR imaging with extended targets, which ensures that the weak targets will not be submerged by the ambiguity noise from channel interference. Second, the configuration of antenna array in MIMO SAR system should be set up properly, which involves the key factors of system performance, including system complexity and system implementation ability. The setup of array antenna configuration also needs to refer to its application, such as HRWS remote sensing, threedimensional (3D) imaging, ground moving target indication (GMTI), and interferometric SAR. Third, the design and realization of signal processing algorithms should be paid attention as well, such as multiple channel filtering, timefrequency processing, and advance digital beam forming (DBF).

How to overcome these challenges is currently the key work of researchers and engineers of MIMO SAR. In this paper, simulations and experiments mainly focus on point targets. Further research work will be around airborne MIMO SAR system design and experiment to study the scenarios of extended targets.

\section{Conclusion}

In this paper, the principle of MIMO SAR using orthogonal coding waveforms is analyzed and imaging algorithm is deduced. For MIMO SAR system, the azimuth ambiguity caused by the PRF reduction is eliminated by the usage of DPCA technique which can increase azimuth sampling rate 


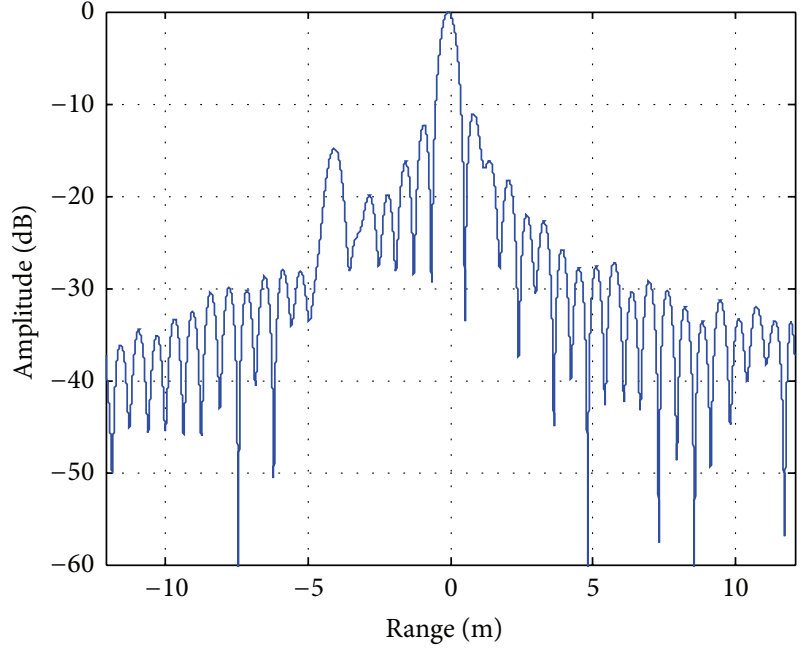

(a) Range slice of imaging result

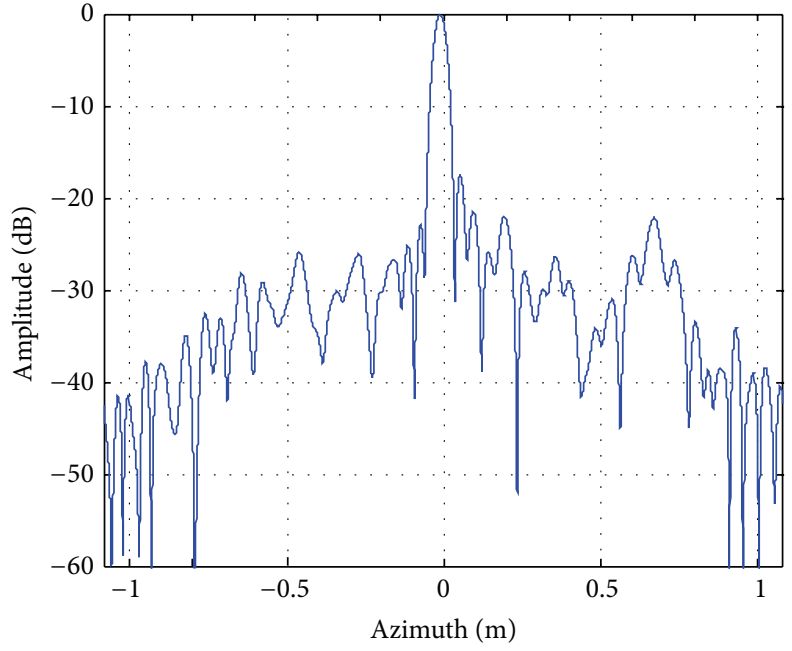

(b) Azimuth slice of imaging result

FIGURE 9: MIMO SAR imaging results.

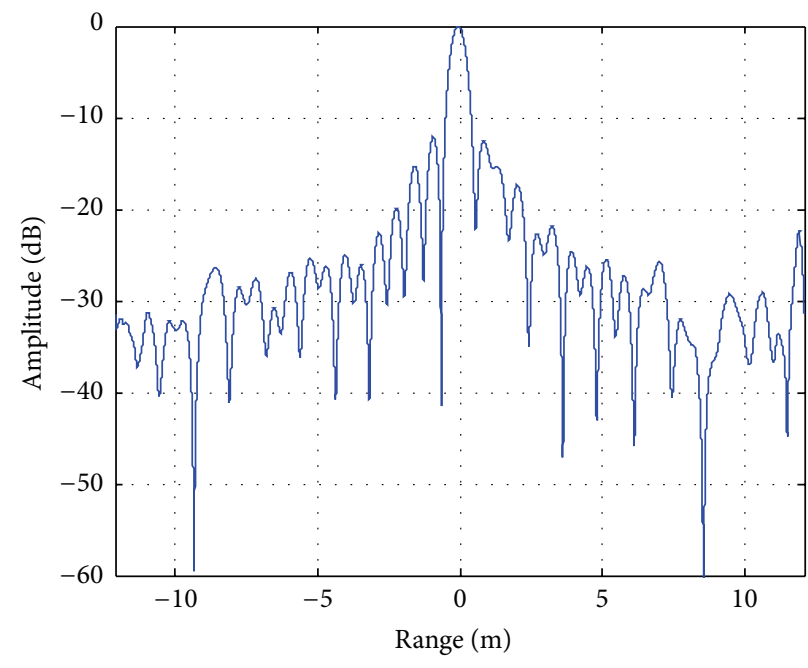

(a) Range slice of imaging result

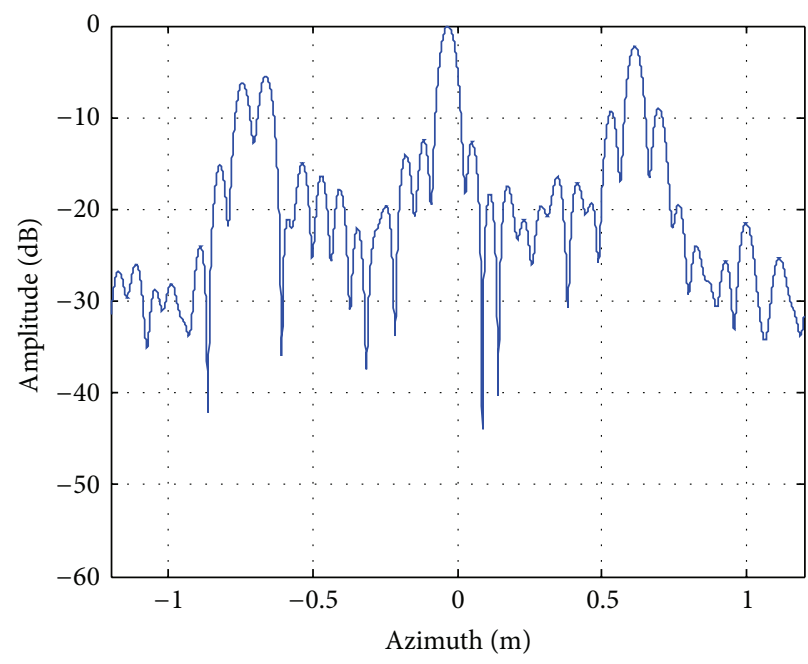

(b) Azimuth slice of imaging result

FIgURE 10: Traditional SISO SAR imaging result.

by $2 N-1$ times. By using orthogonal coding waveform, lower channel interference is obtained, which makes MIMO SAR achieve wider unambiguous range swath and lower azimuth ambiguity. The proposed MIMO SAR can effectively achieve high resolution and wide swath at the same time. For restrictions between swath width and azimuth resolution, formula derivation and data simulation are carried out. Ground-based MIMO SAR system is designed and experiments are carried out, and the performance comparison between SISO SAR and MIMO SAR imaging results validates the practicability and advantage of MIMO SAR system.

\section{Conflict of Interests}

The authors declare that there is no conflict of interests regarding the publication of this paper.

\section{Acknowledgments}

This work is supported by the National High Technology Research and Development Program of China under Grant 2012AA121305 and Natural Science Foundation of Zhejiang province under Grant LQ14D060001.

\section{References}

[1] K. Tomiyasu, "Conceptual performance of a satellite borne wide swath synthetic aperture radar," IEEE Transactions on Geoscience and Remote Sensing, vol. 19, no. 2, pp. 108-116, 1981.

[2] W. Carrara, R. Goodman, and R. Majewski, Spotlight Synthetic Aperture Radar: Signal Processing Algorithms, Artech House, Boston, Mass, USA, 1995.

[3] A. Currie and M. A. Brown, "Wide-swath SAR," IEE Proceedings of Radar, Sonar and Navigation, vol. 139, no. 2, pp. 122-135, 1992. 
[4] S. Li, W. Xu, and P. Huang, "Spaceborne squinted multichannel synthetic aperture radar data focusing," IET Radar, Sonar \& Navigation, vol. 8, no. 9, pp. 1073-1080, 2014.

[5] F. Bordoni, M. Younis, E. M. Varona et al., "Performance investigation on scan-on-receive and adaptive digital beamforming for high-resolution wide-swath synthetic aperture radar," in Proceedings of the International ITG Workshop Smart Antennas (WSA '09), Berlin, Germany, 2009.

[6] N. Gebert, G. Krieger, and M. A. Moreira, "Digital beamforming on receive: techniques and optimization strategies for highresolution wide-swath SAR imaging," IEEE Transactions on Aerospace and Electronic Systems, vol. 45, no. 2, pp. 564-592, 2009.

[7] P. Huang, W. Xu, and W. Qi, "Two dimension digital beamforming preprocessing in multibeam Scansar," Progress in Electromagnetics Research, vol. 136, pp. 495-508, 2013.

[8] G. Krieger, N. Gebert, and A. Moreira, "Multidimensional waveform encoding: a new digital beamforming technique for synthetic aperture radar remote sensing," IEEE Transactions on Geoscience and Remote Sensing, vol. 46, no. 1, pp. 31-46, 2008.

[9] E. Fishler, A. Haimovich, R. S. Blum, D. Chizhik, L. Cimini, and R. Valenzuela, "MIMO radar: an idea whose time has come," in Proceedings of the IEEE Radar Conference, pp. 71-78, IEEE, Philadelphia, Pa, USA, April 2004.

[10] L. B. White and P. S. Ray, "Signal design for MIMO diversity systems," in Proceedings of the 38th Asilomar Conference on Signals, Systems and Computers, pp. 973-977, Pacific Grove, Calif, USA, November 2004.

[11] V. F. Mecca, D. Ramakrishnan, and J. L. Krolik, "MIMO radar space-time adaptive processing for multipath clutter mitigation," in Proceedings of the 4th IEEE Sensor Array and Multichannel Signal Processing Workshop, pp. 249-253, Waltham, Mass, USA, July 2006.

[12] J. H. G. Ender, "MIMO SAR," in Proceedings of the International Radar Symposium, no. 9, pp. 580-588, Cologne, Germany, 2007.

[13] J. Kim and A. Ossowska, "Investigation of MIMO SAR for interferometry," in Proceedings of the 4th European Radar Conference, pp. 51-54, Munich, Germany, 2007.

[14] N. Gebert, G. Krieger, and A. Moreira, "Digital beamforming for HRWS-SAR imaging: system design, performance and optimization strategies," in Proceedings of the IEEE International Conference on Geoscience and Remote Sensing Symposium (IGARSS '06), pp. 1836-1839, IEEE, Denver, Colo, USA, JulyApril 2006.

[15] B. Zou, Z. Dong, and D. Liang, "Design and performance analysis of orthogonal coding signal in MIMO-SAR," Science China: Information Sciences, vol. 54, no. 8, pp. 1723-1737, 2011.

[16] W.-Q. Wang, "MIMO SAR imaging: potential and challenges," IEEE Aerospace and Electronic Systems Magazine, vol. 28, no. 8, pp. 18-23, 2013.

[17] W.-Q. Wang, "Space-time coding MIMO-OFDM SAR for high-resolution imaging," IEEE Transactions on Geoscience and Remote Sensing, vol. 49, no. 8, pp. 3094-3104, 2011.

[18] C. Z. Meng, J. Xu, X. G. Xia et al., "MIMO-SAR waveform separation based on inter-pulse phase modulation and rangeDoppler decouple filtering," Electronics Letters, vol. 49, no. 6, pp. 415-417, 2013.

[19] Y. Yang and R. S. Blum, "MIMO radar waveform design based on mutual information and minimum mean-square error estimation," IEEE Transactions on Aerospace and Electronic Systems, vol. 43, no. 1, pp. 330-343, 2007.

[20] M. A. Sebt, A. Sheikhi, and M. M. Nayebi, "Orthogonal frequency-division multiplexing radar signal design with optimised ambiguity function and low peak-to-average power ratio," IET Radar, Sonar and Navigation, vol. 3, no. 2, pp. 122 132, 2009.

[21] P. Huang, H. Li, S. Zhang, and G. Sun, "Joint two-dimensional ambiguity resolving based on space-time filtering for MIMOSAR," International Journal of Antennas and Propagation, vol. 2014, Article ID 452141, 8 pages, 2014. 

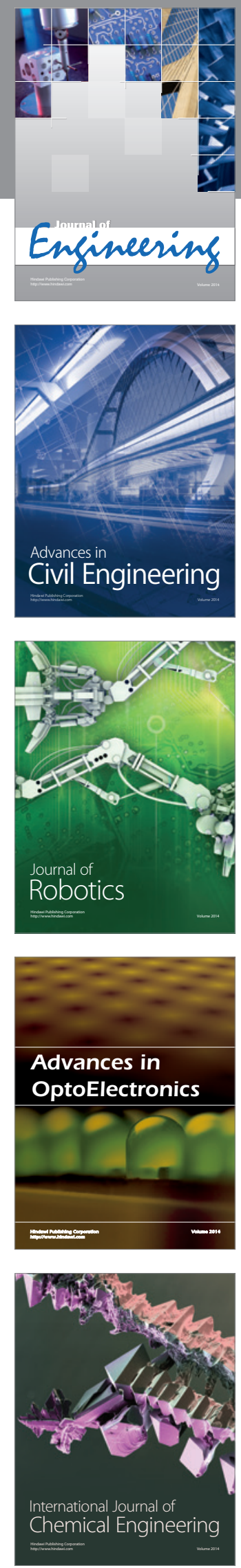

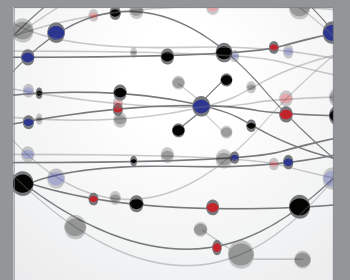

The Scientific World Journal
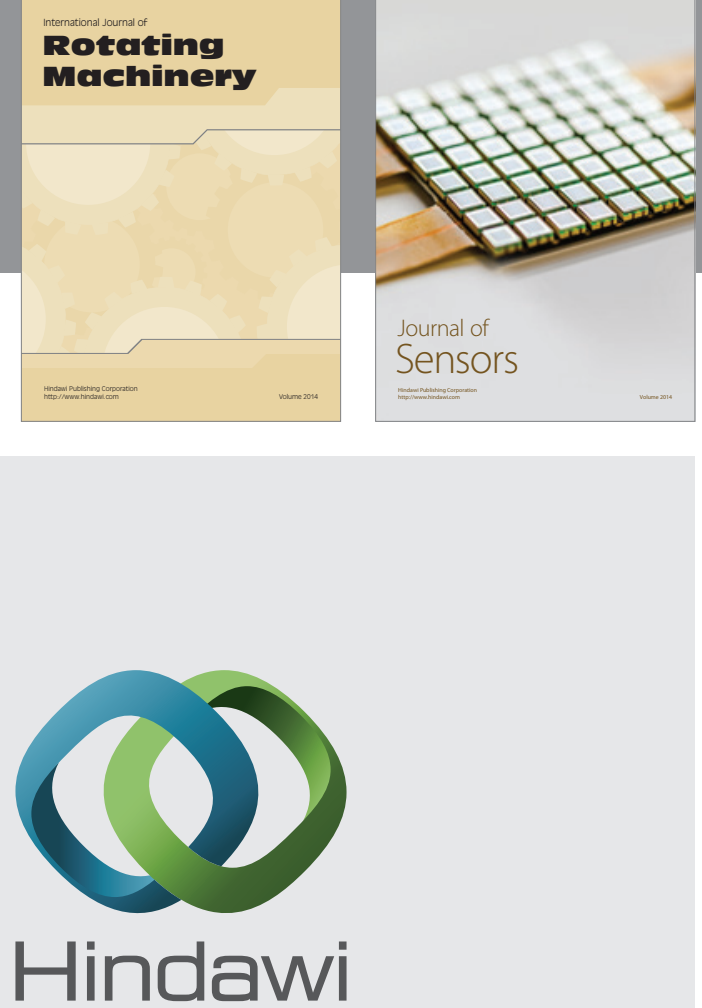

Submit your manuscripts at http://www.hindawi.com
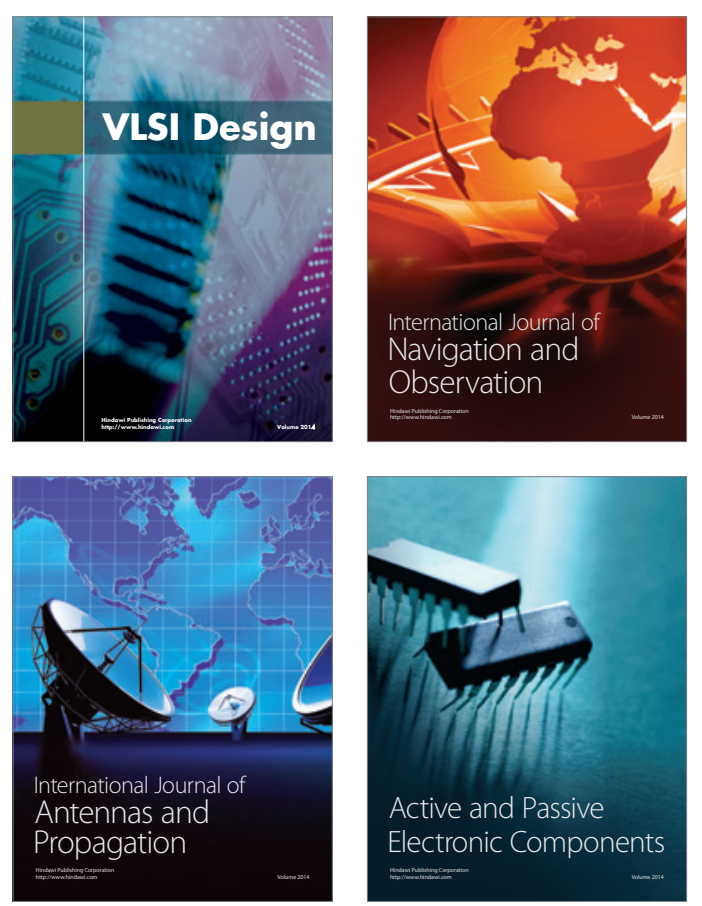
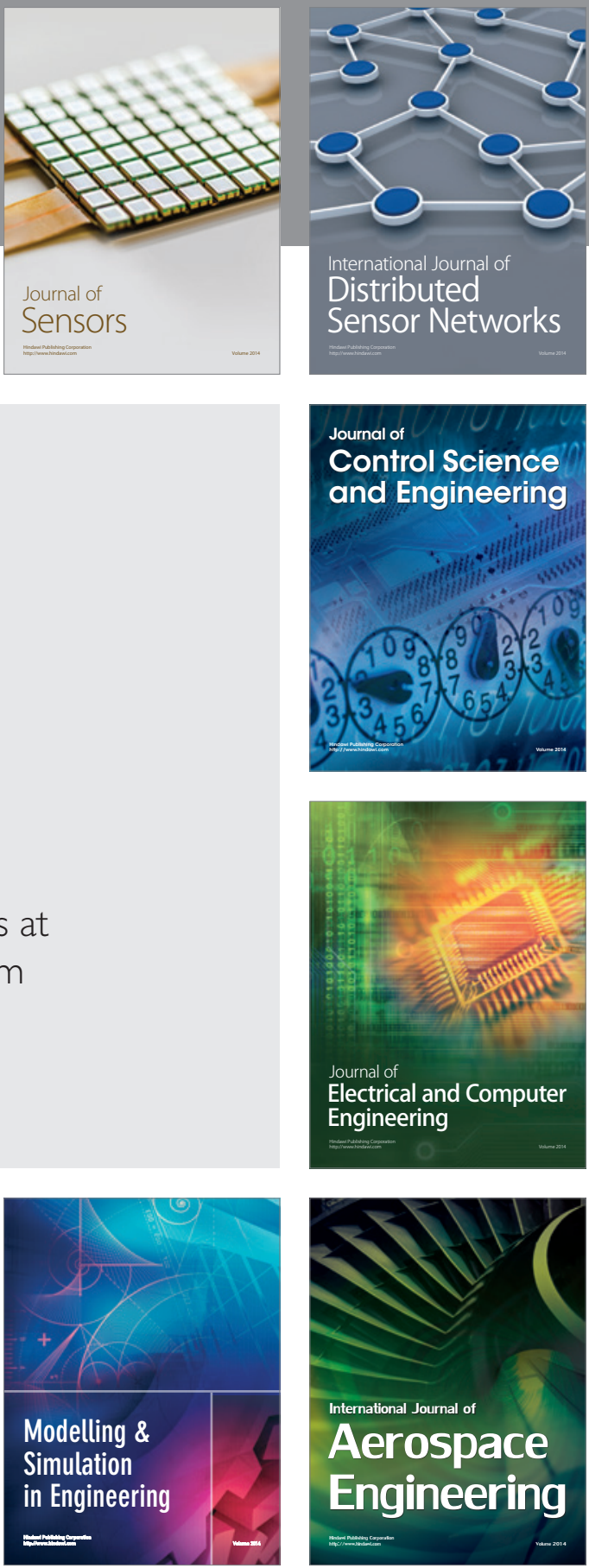

Journal of

Control Science

and Engineering
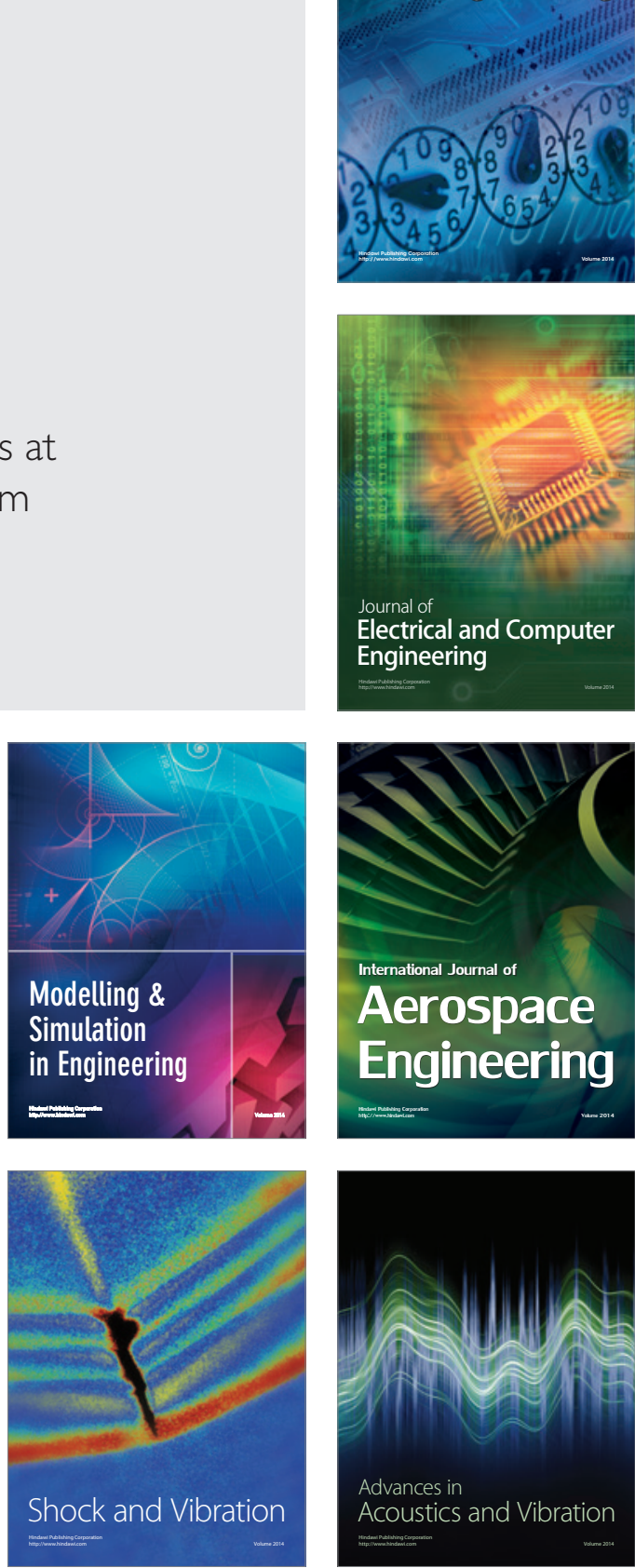